\title{
Prediction of vulnerability to bipolar disorder using multivariate neurocognitive patterns: a pilot study
}

\author{
Mon-Ju Wu ${ }^{1,3^{*+}} \odot$, Benson Mwangi ${ }^{1 \dagger}$, Ives Cavalcante Passos ${ }^{1}$, Isabelle E. Bauer ${ }^{1}$, Bo Cao ${ }^{1}$, Thomas W. Frazier ${ }^{2}$, \\ Giovana B. Zunta-Soares ${ }^{1}$ and Jair C. Soares ${ }^{1}$
}

\begin{abstract}
Bipolar disorder (BD) is a common disorder with high reoccurrence rate in general population. It is critical to have objective biomarkers to identify BD patients at an individual level. Neurocognitive signatures including affective Go/ No-go task and Cambridge Gambling task showed the potential to distinguish BD patients from health controls as well as identify individual siblings of BD patients. Moreover, these neurocognitive signatures showed the ability to be replicated at two independent cohorts which indicates the possibility for generalization. Future studies will examine the possibility of combining neurocognitive data with other biological data to develop more accurate signatures.
\end{abstract}

Keywords: Bipolar disorder, Neurocognition, Vulnerability, CANTAB, Machine learning

\section{Correspondence}

Bipolar disorder (BD) has a lifetime prevalence of $4-5 \%$ in the general population. It is frequently associated with high rates of morbidity, mortality, and completed suicides (Mathers et al. 2006; Merikangas 2007; Nordentoft et al. 2011). It has been reported that only $20 \%$ of BD patients experiencing a depressive episode are diagnosed with $\mathrm{BD}$ within the first year of seeking treatment. This greatly underscores the need for objective diagnostic and vulnerability markers of this debilitating illness (Goldberg et al. 2001). Noticeably, previous epidemiological studies have reported that first-degree relatives of $\mathrm{BD}$ patients have an increased tenfold risk of $\mathrm{BD}$ as compared to the general population-which strongly highlights the role of genetic factors to the etiology of BD (Kessler et al. 1994; Olvet et al. 2013). However, despite these facts, there are no clinically useful biomarkers of vulnerability to $\mathrm{BD}$ that guides the institution of prophylactic interventions. These timely interventions may delay the onset

\footnotetext{
${ }^{*}$ Correspondence: mon-ju.wu@uth.tmc.edu

${ }^{\dagger}$ Mon-Ju Wu and Benson Mwangi contributed equally to this work

${ }^{3}$ Department of Psychiatry \& Behavioral Sciences, The University of Texas

Health Science Center, 1941 East Road, Houston, TX 77054, USA

Full list of author information is available at the end of the article
}

of $\mathrm{BD}$ and translate into better clinical outcomes such as decreased rates of recurrence, less severe episodes (Post et al. 2010), and reduced medical related costs due to less hospitalizations.

Multiple studies have reported neurocognitive abnormalities in BD patients as compared to demographically matched healthy controls (HCs). These abnormalities have primarily been shown in key cognitive domains such as: executive function, sustained attention, verbal learning, and working memory (Robinson and Ferrier 2006; Torres et al. 2007; Arts et al. 2008; Bora et al. 2009; Torres et al. 2010; Mann-Wrobel et al. 2011; Bourne et al. 2013; Bauer et al. 2015; Wu et al. 2016). Furthermore, studies examining neurocognitive measurements in firstdegree relatives of $\mathrm{BD}$ patients have also reported deficits in unaffected first-degree relatives in similar neurocognitive domains. A recent meta-analysis summarized studies investigating neurocognitive endophenotypes in BD and reported abnormalities in first-degree relatives of BD patients in key domains such as: set-shifting, processing speed, verbal learning, and response inhibition (Bora et al. 2009). Similarly, in a recent review, Olvet et al. reported a consistent theme on memory-related deficits in unaffected twins and siblings of patients with BD as compared to HCs (Olvet et al. 2013). Specifically, 


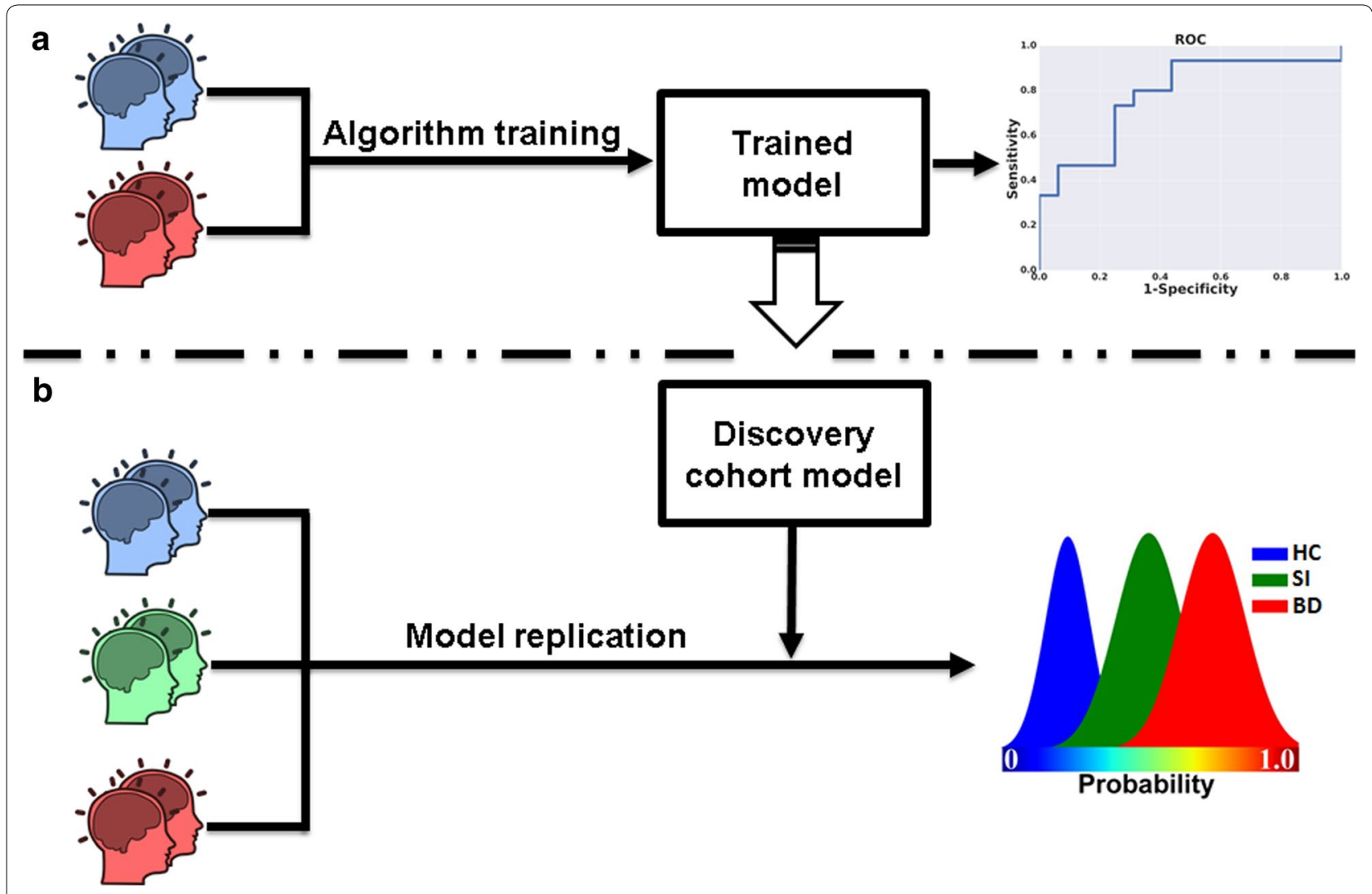

Fig. 1 A flow diagram showing the signature discovery and replication stages

verbal, declarative, and working memory deficits were shown in unaffected siblings (Gourovitch et al. 1999; Kéri et al. 2001; Kieseppä et al. 2005; Christensen et al. 2006). Moreover, several other studies have highlighted executive function and verbal memory abnormalities as candidate endophenotypes of BD following reported deficits in these domains in first-degree relatives of BD patients (Arts et al. 2008; Bora et al. 2009; Doyle et al. 2009). However, while these studies have undeniably advanced our understanding of vulnerability markers of BD, it remains unknown whether reported abnormalities can objectively identify unaffected individuals vulnerable to BD and at an individual level. Noticeably, being able to predict an individual participant's probability of vulnerability to BD based on a hazard-free and easily accessible neurocognitive task could help in institution of individualized prophylactic interventions and translate into favorable clinical outcomes.

To achieve this objective, we recruited 21 euthymic BD patients (7 males, 14 females; age: $36.12 \pm 16.55$ years) and 21 demographically matched HCs (5 males, 16 females; age: $36.08 \pm 12.66$ years) at the University of North Carolina at Chapel Hill-a sample we refer to as the discovery cohort. A set of neurocognitive task scores were assessed for each individual using the Cambridge neuropsychological test automated battery (CAN$\mathrm{TAB})$. The nine assessed CANTAB neurocognitive tasks include: Affective Go/No-Go, Big/Little Circle, Cambridge Gambling Task, Choice Reaction Time, Motor Screening, Match to Sample Visual Search, Rapid Visual Processing, Spatial Recognition Memory, and Spatial Span task. The essence and measurements of all nine tasks are summarized in Table 1. As a second step, a replication cohort of $15 \mathrm{BD}$ patients ( 5 males, 10 females; age: $32.67 \pm 9.26$ years) and 16 demographically matched HCs (5 males, 11 females; age: $33.75 \pm 10.95$ years) were assessed at the University of Texas Health Science Center at Houston. A set of CANTAB neurocognitive task measurements similar to the discovery cohort was also assessed. Notably, in the second center (replication cohort), an additional group of 15 age- and gender-matched siblings (SI) (4 males, 11 females; age: $32.20 \pm 11.69$ years) of BD patients (non-affected with $\mathrm{BD})$ were also recruited and their CANTAB measurements were assessed. These data were first used to 'train' a least absolute shrinkage selection operator (LASSO) machine-learning algorithm in distinguishing patients from HCs. Second, the established predictive signature 

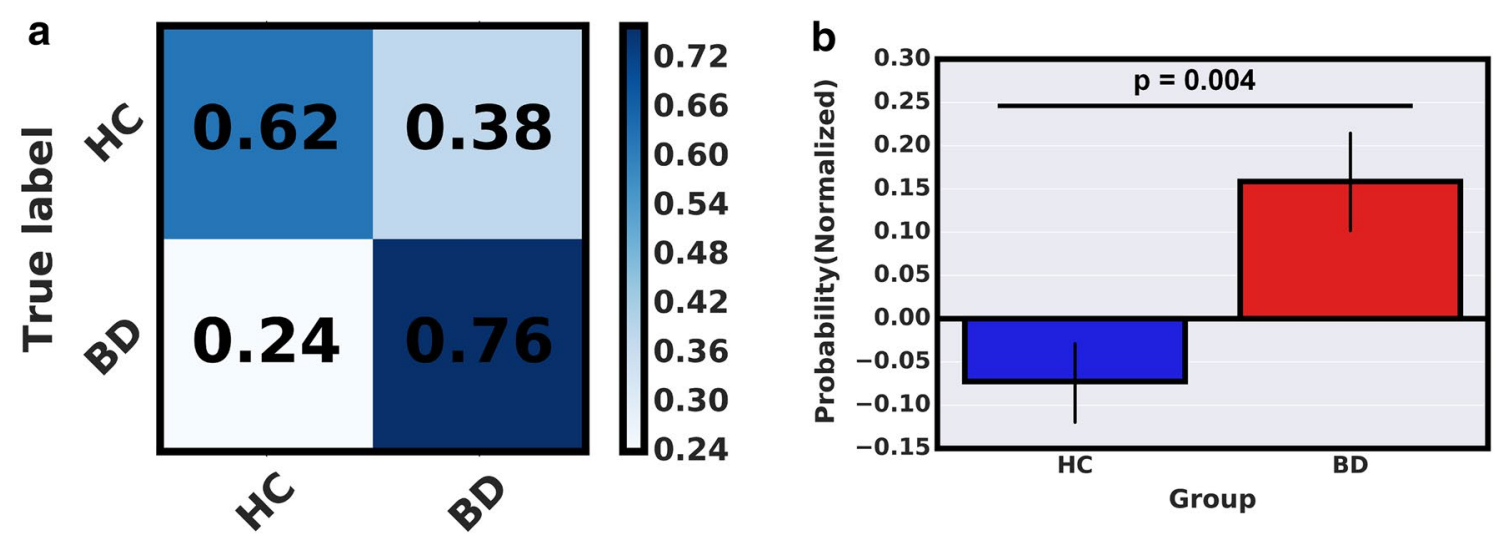

\section{Predicted label}

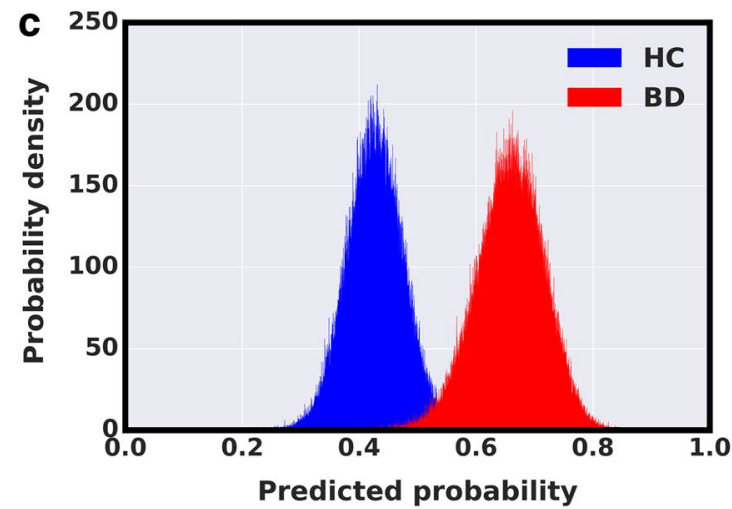

Fig. 2 a A'confusion matrix' depicting actual and LASSO predicted diagnostic labels in the discovery cohort. $\mathbf{b}$ A comparison of predicted probability scores between BD patients and HCs in the discovery cohort. $\mathbf{c}$ A bootstrapping calculation was performed to estimate distribution of the mean predicted probability for BD patients and HCs in the discovery cohort

was further validated using an independent replication cohort of BD patients and HCs (Fig. 1). Lastly, the extent to which the validated predictive neurocognitive signature may differentiate the siblings (SIs) from HCs and BD patients was also examined.

The LASSO algorithm identified individual BD patients from HCs in the discovery cohort with $69 \%$ accuracy, $76 \%$ sensitivity, $62 \%$ specificity, $67 \%$ of positive predictive values (PPV), 72\% of negative predictive values (NPV), and an area under receiver operating characteristic curve (AUROC) of 0.6905 with Chi-square $p=0.0126$ (Fig. 2 and Additional file 1: Table S1). In the discovery cohort, predictor variables identified by the LASSO algorithm as most relevant in distinguishing BD patients from $\mathrm{HCs}$ (non-zero coefficients) include: number of omission errors to negative stimuli on the Affective Go/No-Go task, delay aversion, and the risk adjustment on the Cambridge Gambling Task and the total number of hits on the Rapid Visual Processing (Fig. 3 and Additional file 1: Table S2). In the replication cohort, the LASSO model derived at the discovery stage identified individual BD patients from HCs in the replication cohort with $74 \%$ accuracy, $73 \%$ sensitivity, $75 \%$ specificity, $73 \%$ of PPV, $75 \%$ of NPV, and an AUROC of 0.7417 (Fig. 4 and Additional file 1: Table S3). These predictions were significant (Chi-square $p=0.007$ ). Predicted probability scores of HCs differed significantly from SIs and BD patients with $p=0.027$ and $p=0.008$, respectively. On the other hand, SIs were largely indistinguishable from BD patients with $p=0.678$. These tests were performed using a non-parametric Kruskal-Wallis statistical test.

From a cognitive viewpoint, compared to $\mathrm{HCs}$, individuals with $\mathrm{BD}$ committed a greater number of errors when exposed to negative stimuli. This finding provides further support for the presence of a negative affective bias which is reflected by impaired cognitive processing resulting from exposure to negative stimuli in both adults with BD and offspring of BD patients (Pavuluri and Passarotti 2008; Abe et al. 2011; Passarotti et al. 2011, 2012; Bauer et al. 2015). Furthermore, HCs had a higher quality of risk adjustment on the CGT task compared with individuals with BD (Quraishi and Frangou 2002), which is a reliable estimate of impulsivity and risk taking (Swann 
Table 1 Cognitive tasks and measurements

\begin{tabular}{llll}
\hline No. & CANTAB task & Evaluation & Measurements \\
\hline 1 & Affective Go/No-Go & Inhibition control & Comprehension, learning and reversal \\
2 & Big/Little Circle & Reaction time*, accuracy \\
3 & Cambridge Gambling Task & & $\begin{array}{l}\text { Reaction time*, accuracy } \\
\text { Reaction time*, accuracy, proportion bets } \\
\text { across trials with more/equally/less likely } \\
\text { outcome }\end{array}$ \\
& & Simple (motor) processing speed & Reaction time*, accuracy \\
5 & Choice Reaction Time & Simple (motor) processing speed & Reaction time* \\
6 & Motor Screening & Ability to match motor and visual stimuli & Reaction time*, accuracy \\
7 & Match to Sample Visual Search & Sustained attention & Reaction time*, accuracy \\
8 & Rapid Visual Processing & Visual spatial recognition memory & Reaction time*, accuracy \\
9 & Spatial Recognition Memory & Spatial working memory & Span length, number of attempts, reaction \\
\end{tabular}

*Reaction time is in milliseconds

et al. 2003). Therefore, our findings are consistent with previous evidence that patients with $\mathrm{BD}$ have a high reward-seeking response and are unable to delay gratification (Najt et al. 2007; Swann et al. 2009). Moreover, in spite of the absence of a diagnosis of $\mathrm{BD}$, the at-risk individuals displayed the tendency to make poorer decisions compared with HCs. This finding is particularly relevant because, to date, few studies have focused on the cognitive functioning of siblings of BD patients. Previous studies of unaffected siblings found that they scored
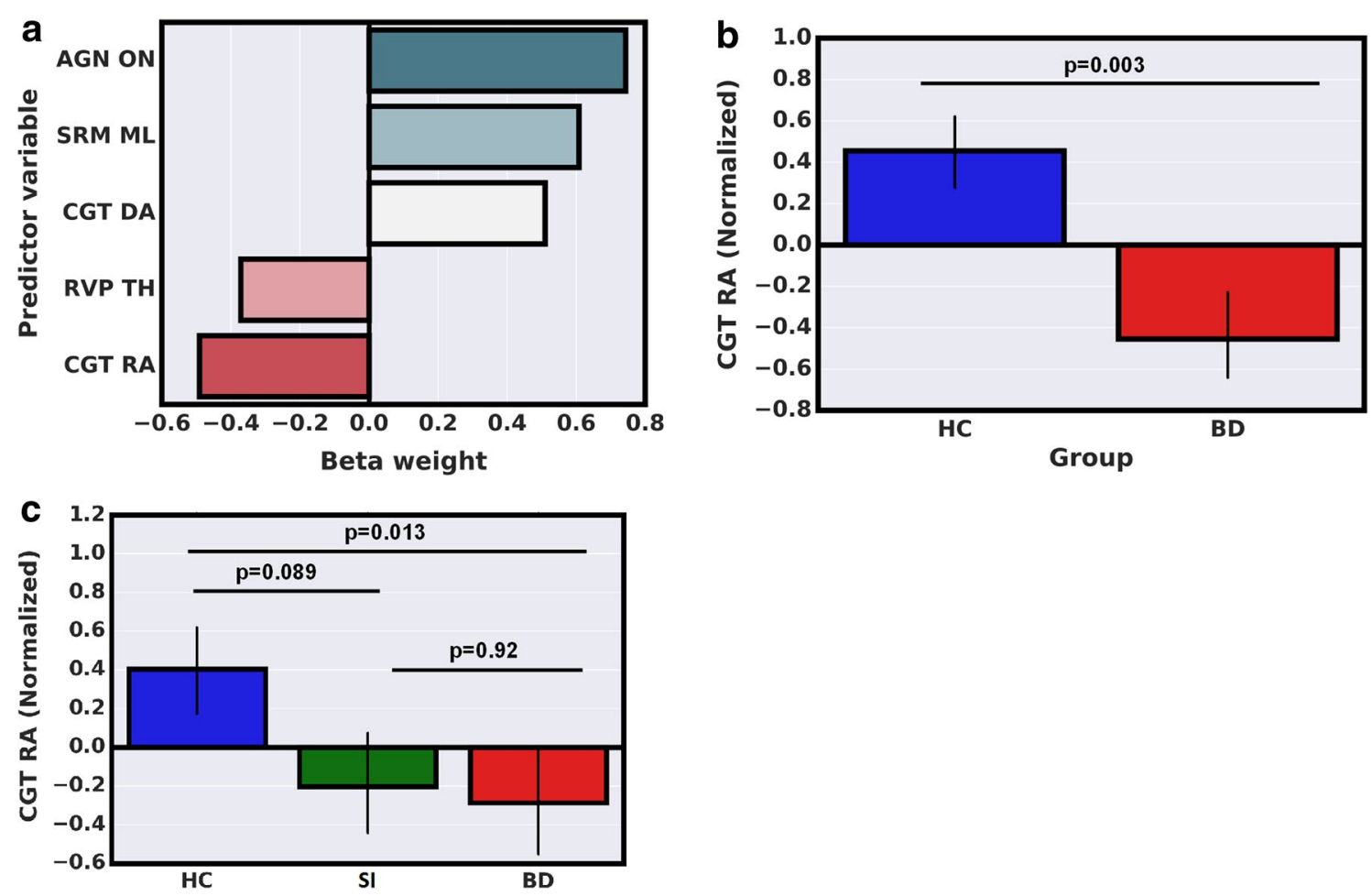

Fig. 3 a A bar graph showing LASSO algorithm coefficients assigned to the most relevant CANTAB neurocognitive measurements. AGN ON affective Go/No go task total omission with negative stimuli, SRM ML spatial recognition memory task mean latency, CGT DA Cambridge gambling task delay aversion, CGT RA Cambridge gambling task risk adjustment, RVP TH rapid visual processing task total hits. These neurocognitive variables were assigned as non-zero coefficients during algorithm training. Positive coefficients represent increased neurocognitive scores in BD patients as compared to HCs and vice versa. b A bar graph comparing CGT RA scores from the discovery cohort. c A three-group (HCs, Sls, BD) comparison of CGT RA scores in the replication cohort 

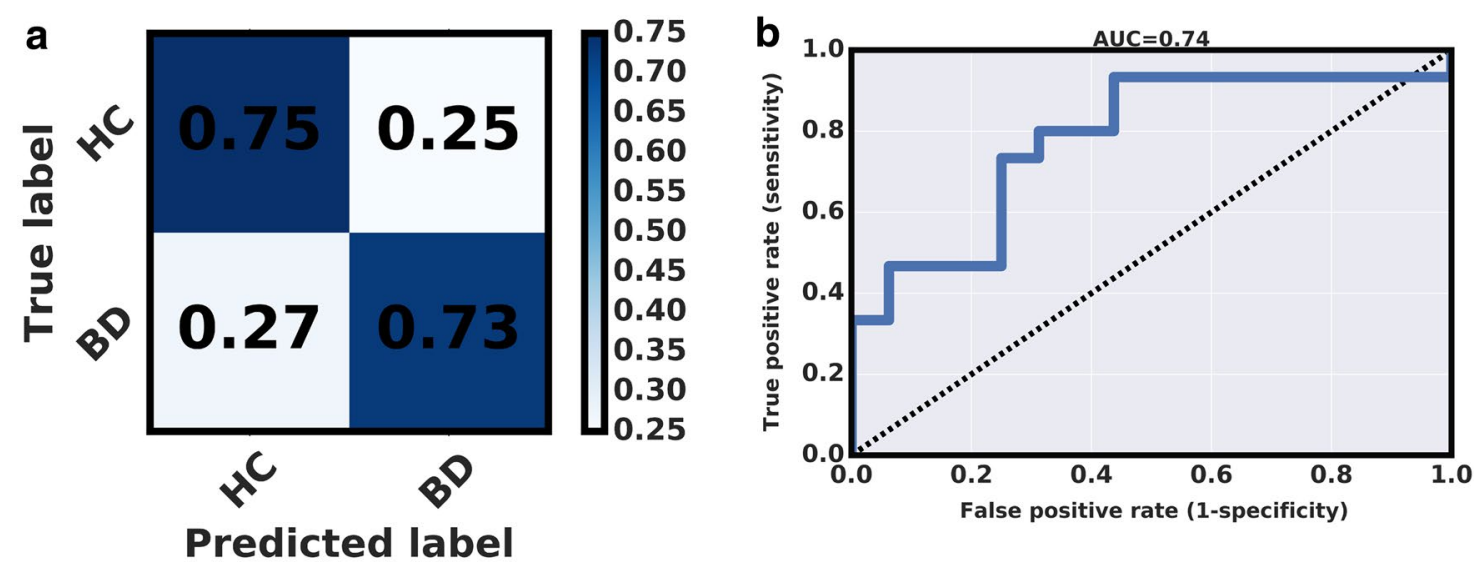

C

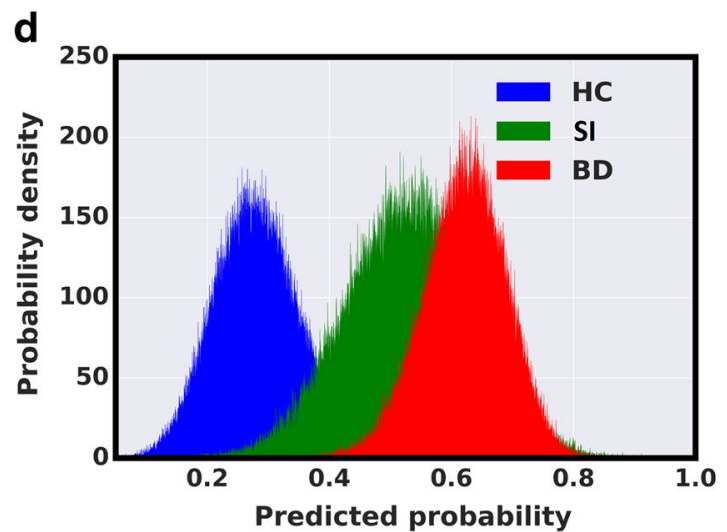

Fig. 4 a A'confusion matrix' representing actual and predicted patient and HCs labels in the replication cohort. b A receiver operating characteristic (ROC) curve depicting the algorithm's performance in distinguishing BD patients from HCs in the replication cohort. $\mathbf{c} A$ bar graph comparing predicted probability scores between HCs, Sls, and BD patients HCs in the replication cohort. $\mathbf{d}$ A bootstrapping calculation was used to estimate the distribution of the mean predicted probability scores for BD patients, SIs, and HCs

lower on tests of verbal learning, attention, and planning than healthy individuals (Kéri et al. 2001; Trivedi et al. 2008; Kulkarni et al. 2010; Nehra et al. 2014). Further, in line with our findings, the magnitude of these cognitive deficits of SIs has consistently been reported to be intermediate between that of $\mathrm{HCs}$ and BD patients. Another potential implication of our findings is that impulsivity, a trait typically associated with BD (Newman and Meyer 2014) and underlying decision making and reward tasks (Christodoulou et al. 2006) is a potential marker of vulnerability to BD in SIs.

The current study has some potential limitations. The overall sample size in both discovery and replication cohorts were small and therefore our results should be regarded as preliminary. The discovery cohort was relatively small as we only considered euthymic patients at the signature discovery stage to avoid potential confounders related to mood phase (e.g., depression, mania).
Six SI participants were diagnosed with other mood disorders other than BD (e.g., major depression) and future studies should examine this research question using an SI cohort without any psychiatric diagnoses. BD patients included in the discovery cohort were taking psychotropic medications which may be a potential confounder but also a reflection of standard clinical practice.

In conclusion, we report a study showing neurocognitive signature able to distinguish individual BD patients from HCs. We suggest this signature could be combined with other biological features to potentially develop a BD prediction model. However, the current study serves as a proof-of-concept. Future studies will examine this hypothesis using other biological markers (e.g., neuroimaging) as well as attempt to integrate multi-scale biomarkers (e.g., neuroimaging and neurocognition) which may potentially improve the current prediction results. 


\section{Additional file}

Additional file 1. Detailed prediction results in both discovery and replication cohorts.

\section{Publisher's Note}

Springer Nature remains neutral with regard to jurisdictional claims in published maps and institutional affiliations.

Received: 24 November 2016 Accepted: 19 July 2017

Published online: 01 September 2017

\begin{abstract}
Abbreviations
BD: bipolar disorders; HC: healthy control; SI: sibling; CANTAB: Cambridge Neurocognitive Test Automated Battery; UNC: University of North Carolina at Chapel Hill; UTHealth: University of Texas Health Science Center at Houston; HAMD: Hamilton Depression Rating Scale; MADRS: Montgomery-Åsberg Depression Rating Scale; YMRS: Young Mania Rating Scale; LASSO: Least Absolute Selection Shrinkage Algorithm; PPV: positive predictive values; NPV negative predictive values; AUROC: area under receiver operating characteristic curve.
\end{abstract}

\section{Authors' contributions}

MW was in charge of data preprocessing, implementation of machine learning algorithms, data interpretation, and manuscript preparation. BM participated in the implementation of machine learning algorithms, data interpretation, and manuscript preparation. IP participated in data preprocessing, data interpretation, and manuscript preparation. IB participated in data preprocessing, data interpretation, and manuscript preparation. BC participated in data interpretation and manuscript preparation. TF participated in data acquisition, data interpretation, and manuscript preparation. GZ participated in data acquisition, data interpretation, and manuscript preparation. JS participated in data acquisition, data interpretation, and manuscript preparation. All authors read and approved the final manuscript.

\section{Author details}

${ }^{1}$ UT Center of Excellence on Mood Disorder, Department of Psychiatry and Behavioral Sciences, The University of Texas Science Center at Houston, Houston, TX, USA. ${ }^{2}$ Cleveland Clinic Children's Hospital Center for Pediatric Behavioral Health, Cleveland, OH, USA. ${ }^{3}$ Department of Psychiatry \& Behavioral Sciences, The University of Texas Health Science Center, 1941 East Road, Houston, TX 77054, USA

\section{Acknowledgements \\ Not applicable.}

\section{Competing interests}

Prof. Soares has participated in research funded by Forest, Merck, BMS, GSK and has been a speaker for Pfizer and Abbott. Dr. Frazier has received federal funding or research support from, acted as a consultant to, received travel support from, and/or received a speaker's honorarium from the Simons Foundation, the Ingalls Foundation, Forest Laboratories, Ecoeos, IntegraGen, Kugona LLC, Shire Development, Bristol-Myers Squibb, the National Institutes of Health, and the Brain and Behavior Research Foundation. All other authors have no interests to declare.

\section{Availability of data and materials}

All data were stored at secured places at the University of Texas Health Science Center at Houston and can only be accessed by authorized personnel under the supervision of IRB.

\section{Consent for publication}

All author gave their consent to publish this manuscript.

\section{Ethics approval and consent to participate}

This study was approved by the University of North Carolina at Chapel Hill (UNC) and the University of Texas Health Science Center at Houston (UTHealth) Institutional Review Boards. All participants signed informed consent before any study-related procedures were performed.

\section{Funding}

This research was funded by NIH R01 MH 085667, the Dunn Foundation and Pat Rutherford, Jr. Chair in Psychiatry at UTHealth to Jair C. Soares.

\section{References}

Abe N, Uchida S, Otsuki K, Hobara T, Yamagata H, Higuchi F, Shibata T, Watanabe $Y$. Altered sirtuin deacetylase gene expression in patients with a mood disorder. J Psychiatr Res. 2011;45:1106-12.

Arts B, Jabben N, Krabbendam L, van Os J. Meta-analyses of cognitive functioning in euthymic bipolar patients and their first-degree relatives. Psychol Med. 2008;38:771-85.

Bauer IE, Ouyang A, Mwangi B, Sanches M, Zunta-Soares GB, Keefe RSE, Huang $H$, Soares JC. Reduced white matter integrity and verbal fluency impairment in young adults with bipolar disorder: a diffusion tensor imaging study. J Psychiatr Res. 2015;62:115-22.

Bora E, Yucel M, Pantelis C. Cognitive endophenotypes of bipolar disorder: a meta-analysis of neuropsychological deficits in euthymic patients and their first-degree relatives. J Affect Disord. 2009;1 13:1-20.

Bourne C, Aydemir Ö, Balanzá-Martínez V, Bora E, Brissos S, Cavanagh JTO, Clark L, Cubukcuoglu Z, Dias VV, Dittmann S, et al. Neuropsychological testing of cognitive impairment in euthymic bipolar disorder: an individual patient data meta-analysis. Acta Psychiatr Scand. 2013;128:149-62.

Christensen MV, Kyvik KO, Kessing LV. Cognitive function in unaffected twins discordant for affective disorder. Psychol Med. 2006;36:1119-29.

Christodoulou T, Lewis M, Ploubidis GB, Frangou S. The relationship of impulsivity to response inhibition and decision-making in remitted patients with bipolar disorder. Eur Psychiatr. 2006;21:270-3.

Doyle AE, Wozniak J, Wilens TE, Henin A, Seidman LJ, Petty C, Fried R, Gross $L M$, Faraone SV, Biederman J. Neurocognitive impairment in unaffected siblings of youth with bipolar disorder. Psychol Med. 2009;39:1253-63.

Goldberg JF, Harrow M, Whiteside JE. Risk for bipolar illness in patients initially hospitalized for unipolar depression. Am J Psychiatr. 2001;158:1265-70.

Gourovitch ML, Torrey EF, Gold JM, Randolph C, Weinberger DR, Goldberg TE. Neuropsychological performance of monozygotic twins discordant for bipolar disorder. Biol Psychiatr. 1999;45:639-46.

Kéri S, Kelemen O, Benedek G, Janka Z. Different trait markers for schizophrenia and bipolar disorder: a neurocognitive approach. Psychol Med. 2001;31:915-22.

Kessler RC, McGonagle KA, Zhao S, Nelson CB, Hughes M, Eshleman S, Wittchen $\mathrm{H}-\mathrm{U}$, Kendler KS. Lifetime and 12-month prevalence of DSMIII-R psychiatric disorders in the United States: results from the National Comorbidity Survey. Arch Gen Psychiatr. 1994;51:8-19.

Kieseppä T, Tuulio-Henriksson A, Haukka J, Van Erp T, Glahn D, Cannon TD, Partonen T, Kaprio J, Lönnqvist J. Memory and verbal learning functions in twins with bipolar-I disorder, and the role of information-processing speed. Psychol Med. 2005;35:205-15.

Kulkarni S, Jain S, Janardhan Reddy YC, Kumar KJ, Kandavel T. Impairment of verbal learning and memory and executive function in unaffected siblings of probands with bipolar disorder. Bipolar Disord. 2010;12:647-56.

Mann-Wrobel MC, Carreno JT, Dickinson D. Meta-analysis of neuropsychological functioning in euthymic bipolar disorder: an update and investigation of moderator variables. Bipolar Disord. 2011;13:334-42.

Mathers C, Iburg K, Begg S. Adjusting for dependent comorbidity in the calculation of healthy life expectancy. 2006. Popul Health Metr. http:// pophealthmetrics.biomedcentral.com/articles/10.1186/1478-7954-4-4. Accessed 2 Feb 2016.

Merikangas K. Lifetime and 12-month prevalence of bipolar spectrum disorder in the National Comorbidity Survey replication. JAMA Psychiatr. 2007;64(5):543-52.

Najt P, Perez J, Sanches M, Peluso MAM, Glahn D, Soares JC. Impulsivity and bipolar disorder. Eur Neuropsychopharmacol. 2007;17:313-20.

Nehra R, Grover S, Sharma S, Sharma A, Sarkar S. Neuro-cognitive functioning in unaffected siblings of patients with bipolar disorder: comparison with bipolar patients and healthy controls. Indian J Psychiatr. 2014;56:283. 
Newman AL, Meyer TD. Impulsivity: present during euthymia in bipolar disorder? - a systematic review. Int J Bipolar Disord. 2014;2:1-16.

Nordentoft M, Mortensen PB, Pedersen CB. Absolute risk of suicide after first hospital contact in mental disorder. Arch Gen Psychiatr. 2011;68:1058-64.

Olvet DM, Burdick KE, Cornblatt BA. Assessing the potential to use neurocognition to predict who is at risk for developing bipolar disorder: a review of the literature. Cogn Neuropsychiatr. 2013;18:129-45.

Passarotti AM, Ellis J, Wegbreit E, Stevens MC, Pavuluri MN. Reduced functional connectivity of prefrontal regions and amygdala within affect and working memory networks in pediatric bipolar disorder. Brain Connect. 2012:2:320-34.

Passarotti AM, Sweeney JA, Pavuluri MN. Fronto-limbic dysfunction in mania pre-treatment and persistent amygdala over-activity post-treatment in pediatric bipolar disorder. Psychopharmacology. 2011;216:485-99.

Pavuluri MN, Passarotti A. Neural bases of emotional processing in pediatric bipolar disorder. Expert Rev Neurother. 2008;8:1381-7.

Post RM, Leverich GS, Kupka RW, Keck PE, McElroy SL, Altshuler LL, Frye MA, Luckenbaugh DA, Rowe M, Grunze H, et al. Early-onset bipolar disorder and treatment delay are risk factors for poor outcome in adulthood. J Clin Psychiatr. 2010;71:864-72.

Quraishi S, Frangou S. Neuropsychology of bipolar disorder: a review. J Affect Disord. 2002;72:209-26.
Robinson LJ, Ferrier IN. Evolution of cognitive impairment in bipolar disorder: a systematic review of cross-sectional evidence. Bipolar Disord. 2006;8:103-16.

Swann AC, Lijffijt M, Lane SD, Steinberg JL, Moeller FG. Severity of bipolar disorder is associated with impairment of response inhibition. J Affect Disord. 2009;116:30-6.

Swann AC, Pazzaglia P, Nicholls A, Dougherty DM, Moeller FG. Impulsivity and phase of illness in bipolar disorder. J Affect Disord. 2003;73:105-11.

Torres IJ, Boudreau VG, Yatham LN. Neuropsychological functioning in euthymic bipolar disorder: a meta-analysis. Acta Psychiatr Scand. 2007:434:17-26.

Torres IJ, DeFreitas CM, DeFreitas VG, Bond DJ, Kunz M, Honer WG, Lam RW, Yatham LN. Relationship between cognitive functioning and 6-month clinical and functional outcome in patients with first manic episode bipolar I disorder. Psychol Med. 2010;41:971-82.

Trivedi JK, Goel D, Dhyani M, Sharma S, Singh AP, Sinha PK, Tandon R. Neurocognition in first-degree healthy relatives (siblings) of bipolar affective disorder patients. Psychiatr Clin Neurosci. 2008;62:190-6.

Wu M-J, Passos IC, Bauer IE, Lavagnino L, Cao B, Zunta-Soares GB, Kapczinski F, Mwangi B, Soares JC. Individualized identification of euthymic bipolar disorder using the Cambridge Neuropsychological Test Automated Battery (CANTAB) and machine learning. J Affect Disord. 2016:192:219-25.

\section{Submit your manuscript to a SpringerOpen ${ }^{\circ}$ journal and benefit from:}

- Convenient online submission

Rigorous peer review

- Open access: articles freely available online

- High visibility within the field

- Retaining the copyright to your article

Submit your next manuscript at springeropen.com 